\title{
Cloud Migration and Global Digitalization of Business Models
}

\author{
Vasile Mazilescu ${ }^{\star}$
}

\begin{tabular}{l}
\hline \multicolumn{1}{c}{ A R T I C L E I N F O } \\
\hline Article history: \\
Accepted December 2020 \\
Available online December 2020 \\
\hline JEL Classification \\
M10, M15 \\
Keywords: \\
Cloud Computing (CC), Business \\
Models, Digital Platform, \\
Public/Hybrid Cloud (PHC)
\end{tabular}

The economy and business models are evolving extremely fast, being determined by the growing capacity to collect, use and analyze extremely large amounts of data $[9,19]$. These data come from the fingerprints of personal, social activities, as well as from business transactions that take place on various digital platforms based on CC technology $[1,3,18]$. This paper examines how organizations use various cloud architectures and models to increase the internal efficiency of organizations and to create new business services. A lot of digital platforms have emerged around the world using increasingly complex, data-driven business models, disrupting the industries behind them $[12,23,30]$. The strength of the platforms is reflected in the fact that the largest companies in the world are massively implementing platform-based business models. Video conferencing platforms, online shopping and gaming applications have been a symbol of accelerated growth in the last year, and Public Cloud has made possible this extremely rapid transition to smart working and distance education $[4,5]$. The current restrictions on social distancing have led to a huge increase in smart working users and have moved interactions of all kinds into the virtual world. By using PHC technologies, companies have managed to continue their activities, through video conferencing, smart working, distance collaboration in various projects, e-commerce platforms, digital business solutions. Educational continuity could be maintained through e-learning platforms [20]. IaaS providers are not the only major technology players in this period. The sudden transition to online socialization would not be possible without software solutions that allow a fast, secure and consistent distribution of application environments. Various solutions for configuring the cloud infrastructure offer SaaS providers and companies around the world to quickly expand operations with minimal interruptions. The paper presents in section 2 a series of important results and related to the approached topic, as it results from the analyzed literature. Section 3 points out the importance of digitizing services for the economic ecosystem and for other extremely important fields, such as industry, medicine, education. Section 4 analyzes a number of issues that support the migration to the cloud of many classic enterprise-specific computer systems. We argue that this process of cloud migration must be accompanied economically by the change of many business models. The conclusions of the paper are presented in section 5 .

\section{Literature Review}

Digital platforms provide mechanisms for bringing together a set of components to interact online [21]. A distinction can be made between transaction platforms and innovation platforms. Transaction platforms are electronic markets with an online infrastructure that accepts exchanges between several 
different parties. Innovation platforms create environments for code and content producers to develop applications and software. Digital developments have implications for virtually all organizations and will affect all countries, sectors and stakeholders [28,31]. Today, the world is characterized by a gap between unconnected and hyper-digitized countries. The expansion of the digital economy creates many new opportunities for different business economic processes [6,8,16]. Digital data can be used for development purposes and for solving social problems $[7,22]$. Thus, they can contribute to improving economic and social outcomes and can be a force for innovation and productivity growth. The platforms facilitate transactions as well as the exchange of information. From a business perspective, the transformation of all sectors and markets through digitalisation can encourage the production of goods and the provision of high-quality services at low costs $[25,26]$. Digitization transforms value chains in different ways and opens up new channels for added value and wider structural change, but the positive results are far from automatic. Just because digitization has the potential to support development, it is unlikely that any value achieved will be evenly distributed. Even if individuals, companies and countries do not participate in the digital ecosystem, they can be indirectly adversely affected. Workers with limited digital skills will be at a disadvantage compared to those who are better trained, existing local firms will face stiff competition from domestic and foreign digitized ones, and various jobs will be lost due to automation [10,24 ]. The net impact will depend on the level of development and digital availability of countries, on the policies adopted and implemented at national, regional and international level [29]. The impact on value creation and capture can be considered in several economic dimensions (productivity, added value, employment, income and trade), for different actors, micro, small and medium enterprises and for different components of the digital economy.

CC determines change and innovation in production. The rapidly evolving business environment and the need to keep up with the demands of an increasingly diverse consumer base present both challenges and opportunities. For these reasons, many manufacturers use CC as a means of monitoring and managing their processes $[3,11]$. CC has become the main driver of Industry 4.0, in which technologies such as the Internet of Things (IoT), artificial intelligence (AI), blockchain and virtual reality blur the lines between the physical and digital worlds [16]. The ability to connect and transfer data at a desired speed between devices makes it possible for manufacturers, retailers and logistics companies to work together to create solutions to the most pressing business problems [14,17]. CC will affect every aspect of production. From raw material procurement to production and inventory management to delivery, CC is playing an increasingly critical role. Unlike traditional methods of storing and processing where data movement is cumbersome, CC offers manufacturers the flexibility to access data from any location, while increasing security $[13,15]$. Manufacturers, who have already moved to cloud-based systems, consider it financially satisfactory, as the costs of maintaining internal software and IT infrastructure are almost eliminated. With CC, manufacturers can significantly save storage and server costs, resulting in higher performance and cost efficiency. Companies are increasingly using $\mathrm{CC}$ for business innovation to rank first among competitors. The main competitors use the cloud as an important way of communication within the organization. Companies can share knowledge and information between locations in different parts of the globe, so this allows for increased innovation capacity [10]. CC is used by most competitors in decision making. When companies are in such a situation, CC creates an overview of what is happening in the company. Innovation is growing among the main competitors through SaaS, which allows information to be transmitted quickly from one point to another, allows companies to offer new services and change business processes [2]. CC is also used as a way for companies to incorporate and apply mobile technologies, which play an important role in the market because they have an impact on both customers and employees. Cloud provides the necessary infrastructure for new applications and provides companies with ways to incorporate mobile applications with existing systems. CC refers to services shared in institutions through subscription-based models, through which data servers or various applications are accessed, being seen as a transformative technology. This is due to the fact that CC benefits from the rapid implementation of ICT, especially for companies. CC resources can be used to create e-learning platforms, infrastructure and educational services by providing centralized data storage, virtualization and other facilities [27]. All these aspects lead to the need to migrate many activities in the cloud, as well as to the corresponding transformation of many digital business models.

\section{Digital Transformation of Services}

Digital platforms are becoming increasingly important in today's economy. The possible scenarios show two competitive contexts: (i) competition based on the design and management of highly efficient global supply networks in a context of increased uncertainty and instability; (ii) the parallel emergence of local supply chains (at regional, national or continental level) to respond to political, environmental or reliability constraints. In the face of increased competition, innovation is seen as a key point for strong competitiveness. Although the conditions for innovation may be difficult to formalize, its link with research, knowledge management, education and free trade is constantly emphasized. The industrial fabric is mainly composed of small and medium enterprises around the world, being able to disseminate new technologies in small companies and be able to integrate them into global but efficient networks, it is considered a major challenge. The need to have an overall and efficient approach to the life cycle of products and organizations, 
taking into account the three dimensions of sustainable development (economic, social, environmental) is also a common point of most analyzes. The social dimensions of production become a priority (aging of workers and customers, job insecurity, teleworking), so that environmental considerations (ecological design, resource economy) can reorient classic themes to original ones. The perception of increased personalization of products and services as a competitive advantage is universally shared and induces new requirements for the management of information and knowledge in order to develop more complex products. The product will be more active during the manufacturing phase, but also throughout its life cycle, due to environmental intelligence and massively interconnected devices via IoT or IIoT, using emerging technologies. This opens up new perspectives, provided that we can federalize very different communication protocols.

We are witnessing a transition based on technological development from electronic to digital, based on the integration of things (connected and intelligent), with people and businesses. Such an interconnection requires a network that connects everything and a cloud infrastructure that provides all the necessary services to meet any demand for business agility. A vital element in the development of digital business involves capitalizing on the potential of transactional and analytical processing. These characteristics are called digital elements of substance. From a business perspective, there are modeling approaches that provide a language to describe how organizations create, deliver, and capture value. From a technology perspective, we still need a similar minimalist conceptualization, but with a rich semantics, to model digital capabilities. The digital business creates sustained value by being digital connections and using different service-based architectures. Companies connect to other companies, as well as to individuals and assets, while using different types of digital media: web, XML standard, market connectors and so on. Objects are no longer static entities, but rather they become intelligent agents, more and more parts of the world being digitally connected, interacting intelligently with people, businesses. Intelligent agents are typically equipped with sensors that produce data, while even having their own logic of processing and understanding. Smart agents are able to automatically react to contexts without interacting with the customer. The agent category includes robots, autonomous vehicles, drones, etc. and also any technology for digital manufacturing (such as 3D printing). They use digital information to perform services in extremely diverse conditions. Services are standalone applications and not just code that can be called by a client application. Accessing a service requires at least crossing the boundaries that separate the processes, and most likely crossing the network and using cross-domain authentication. Each border to be crossed (process, machine, security) reduces performance, increases complexity and increases the probability of failure. It is very important that they must be recognized and treated in the design phase. Developers and service providers can also be separated geographically, which adds a new boundary, which is reflected in increased development costs and reduced robustness. The answer to these challenges is to keep it simple both in terms of defining the service and in terms of supporting standards in the field of Web services. Good services have a simple interface and share as few abstractions and constraints as possible. Simplicity makes a service easy to use and understand by those who will develop customers for that service.

Service-based architectures and Web services are the latest step in the development of middleware technologies. These technologies solve the problem of interoperability and provide the basis for the development of large Internet applications. Middleware technologies that enable application integration are used for a variety of purposes, from interconnecting the components of a desktop or Web application to developing software systems that run on the Internet. Traditional technologies such as J2EE or messagebased application servers are ideal solutions for developing software systems that run within a single organization. However, they are quite limited when it comes to the interconnection of software systems used by different organizations, which are connected via the Internet. Web services and service-based architectures are designed to meet these needs. In many ways, service-based technologies and Web services are nothing new. Like the rest of the technologies and architectures that support distributed computing, the main purpose of service-based technologies is to support a software system to invoke the functionality offered by another software system (as J2EE allows Java clients to call methods implemented by J2EE components). The main difference is that the emphasis in the case of service-based architectures is on interoperability and problem solving through the use of different platforms and languages. Although a service-based architecture can be developed using any technology that allows distributed computing, only Web services offer an unlimited degree of interoperability. The need for interoperability stems from the fact that most large companies have a mix of software systems in terms of programming languages and platforms used. Given that the re-implementation of these systems on a single platform is much too expensive, it is obvious the need for middleware technologies that allow communication between these software systems. The software systems that need to communicate can be developed for incompatible platforms, so interoperability is needed. Web services and service-based architectures are a solution that allows the integration of different technologies.

Cloud provides a way for businesses to leverage the capabilities supported by these trends and properties of distributed systems to better meet customer needs and drive future growth in digital services. Services are standalone and standalone applications and not classes or components closely related to a particular application [2]. The services are designed to be installed on a network, possibly the Internet, where 
they can be easily integrated into an application where they are needed. The services must know nothing about customers and must accept requests from anywhere, as long as the messages received comply with the format recognized by the service and the security requirements are met. Services can be installed and managed independently of other services and potential client applications, and service owners can change the interface and functionality of a service at any time. Compatibility with previous versions is an important issue for any distributed computing system, especially in the case of web services that are open by definition. The solution to this problem is partly solved by the simplicity and extensibility of a service. All customers know about a service is the kind of messages they accept or return. Thus, a service can be modified as long as previous messages are still accepted as valid messages. Even messages representing requests or replies can be extended, as long as compatibility with previous messages is maintained. As services are standalone applications, they must ensure their security, they must protect themselves against malicious calls. Software systems that are installed on a closed network can largely ignore security issues. In this case it is enough to rely on firewalls or secure communication protocols such as SSL. However, services accessible on the Internet must take much more stringent security measures [24]. The use of microservices in the design and development of applications is a new architectural trend for IT software services. Microservices is a software application development technique that structures an application as a collection of freely coupled services. In the microservice-based architecture, services are broken down into smaller units to improve their modularity. They are developed, tested and implemented as unique application functionality modules. Because they are organized around business processes and are developed independently, they become freely coupled and must be interconnected. The ability of microservices to run independently of each other is used especially in the continuous delivery of scheduled software. Microservices can be scaled independently of other services and can be implemented on the hardware assigned to their needs. Moreover, due to their small size, they are easier to maintain and more error tolerant, because the failure of a service will not interrupt the execution of the entire system, as in the case of a monolithic system.

\section{Adoption of CC type solutions as a business model}

CC makes optimal use of dynamic resource allocation in order to deliver a large number of diverse services to users. Users can focus on application development, outsourcing the running and execution of processes in the system to cloud providers. $\mathrm{CC}$ is a model that allows access to computing resources that have evolved in information technology and has become a dominant business model for the delivery of IT infrastructure, components and applications. With CC, a product-centric model for IT supply is transformed into a global, distributed, service-centric model, leading to a disruptive shift from IT-as-product to IT-asservice. Since its inception in 2007, CC has changed the way IT services are invented, developed, implemented, scaled, upgraded, maintained and paid for. CC allows individuals and organizations to access IT resources on demand, from any device and at any time, as a measured service. They reduce costs in the case of HPC, allowing organizations to use computing power for which they have neither the capital budget nor the necessary operational expertise. While the market is currently demanding the availability of on-demand IT resources, cloud providers are offering an increasing number and variety of services, which are a common base of computing resources and can be flexibly extended to computing increasing. CC also provides the infrastructure that has fueled key digital trends, including mobile computing, IoT (IIoT), big data and Artificial Intelligence.

The main technology behind CC is virtualization. The CC delivery model known as Software as a Service has raised virtualization to the application level, as a business model through which the payment of services is made according to the resources consumed and depending on the type of software application used. The basics of virtualization are represented by the emulation of several computers on the same physical machine. This type of virtualization is known as full virtualization because it allows the installation of an operating system in the isolated framework of a virtual environment. The concept of virtualization is not limited to emulating a computer as a whole. There are many virtualization technologies specific to other types of resources, such as storage media, databases, system memory, network equipment. Each of them adds a layer of abstraction between virtualized resources and physical resources. A cloud-based system can use several virtualization technologies within it, each with specific interfaces defined for virtualized resource management. In recent years, server virtualization has produced a real revolution in the IT field. The traditional approach provided for an application on the server, several applications installed simultaneously can generate crashes. This meant in addition to a very low CPU utilization rate on the server, high power consumption, high maintenance costs, lack of physical space for hardware, and high maintenance costs. In the Cloud model, virtualization is the basic technique for achieving elasticity parameters and widely distributed resources. Just as virtual machines are the building blocks of cloud infrastructure, containers allow for lowerlevel virtualization by composing their features as application packages from individual images. They require low resource and time consumption. Containerization is based on the ability to develop, test and implement applications for operation. Containers are used at the PaaS level in the CC.

Automation of maintenance and operational activities of the services is an important feature for the cloud service provider. Automation refers to cloud resource management, operations, and maintenance 
systems. The resource management process must be fully automated and have a minimum response time. Cloud resources and services need to be constantly monitored to ensure their availability. Various automated utilities are used that can continuously monitor infrastructure, platform and data or business processes. Automatic monitoring is required in the delivery of cloud services at the quality expected by the beneficiary. The IT industry correlates the microservices-based architecture with application container technologies, which simplifies automatic application-level deployment. Containers can be trusted for the construction of such units of architecture based on microservices through the autonomous execution of services at the time of running. In order to benefit from the features of microservice-based architecture, several important issues need to be addressed when designing and implementing the software architecture. These include orchestrating and coordinating services, microservices implementation strategies and data storage. The advantages of implementing microservices in virtual or container environments ensure the scalability of the service, making the systems flexible when using a large number of microservices. Decentralized management and data management allow services to be independent and avoid the need to standardize source code writing in a single programming language. The feature of independent software code delivery, isolation from other services and easy-to-implement and service maintenance functions, as well as scaling functions, make microservices a widely adopted architectural model.

CC has a major impact on many areas of technology and IT architecture. Continuous delivery is part of this change, encouraging fewer errors in production systems, a more involved customer who can provide immediate feedback on the acceptance results of new software features, but also better control, good compliance and high quality of services delivered. Existing models are a must follow to provide cloud applications with high quality of services. Cloud is a service delivery model and changes the nature of IT by shifting control and ownership over IT assets to a provider. This increases some risks and reduces others. To benefit from the cloud, you need to identify and address its risks. Examine each cloud feature to make sure you understand its effects. Each has its advantages and disadvantages. For example, pooling of resources is considered. A private cloud controls its workloads. A public cloud has random tasks. Random workload can affect performance. A risk assessment must be used to determine your own objectives. Success depends on understanding how cloud offerings work. Accept that you may not be a good cloud candidate. Each mix of cloud service and delivery model changes costs, controls, and security, and each model only fits certain tasks. The cloud model chosen depends on security, control and the best cost for a particular company. IaaS tasks usually include IT facilities, hosting services, services, storage, etc. PaaS tasks include application development, data, workflow, security services (simple connection), database management, directory services, etc. SaaS tasks include internet services, blogging, surveys, social networking, knowledge sharing, email, collaboration, productivity tools, resource planning, etc.

Confirm your expectations regarding cloud migration. It is necessary to understand how cloud services and deployment models work to the advantage of the company. Efficiency is the result of greater use of resources and lower management costs. Agility results from the rapid supply of infrastructure. Innovation results from using service performance to find improvements in the cloud. The ability to purchase, deliver, consume and audit cloud services must be assessed. IaaS consumers create, install, monitor and manage platforms for services and applications. PaaS consumers develop, test, deploy, and manage cloud-hosted applications. SaaS offers complete applications over a network. Include multifunctional business and IT representatives. It is necessary to determine how cloud features, services, and deployment models affect current operations. The knowledge and skills of the staff dedicated to these activities will need to be examined.

There are a number of extremely important aspects used as criteria to support business model innovation, called business facilitators: cost flexibility, business scalability, market adaptability, masked complexity, context-based variability and ecosystem connectivity. Cost flexibility is a key reason why many companies are considering adopting the cloud. By adopting cloud services, an organization can transfer costs from fixed to variable. The organization pays for what it needs when it needs it. This pay-per-use model offers greater flexibility and eliminates the need for significant capital expenditures. CC offers more than just IT scalability - it allows an organization to easily scale its business operations. By enabling the rapid supply of resources without scale limitations, the cloud allows a company to benefit from economies of scale without achieving large volumes on its own. Recognizing the ability of the cloud to facilitate efficient business growth, scalability is considered a top advantage for the cloud. In today's economic environment, companies are constantly looking for ways to improve their agility, to adapt to market requirements. By enabling companies to quickly adjust processes, products and services to meet the changing needs of the market, the cloud in turn facilitates rapid prototyping and innovation and helps accelerate time to market. Content and applications from Web content creators, TV networks, advertisers, and other media entities can be developed quickly using standard Web tools. Thanks to its extensive computing power and capability, the cloud can store information about users' preferences, which can allow them to customize their product or service. Contextbased variability, offered through the cloud, allows companies to provide users with personal experiences that adapt to subtle changes in the user-defined context, allowing for a more user-centric experience. Context-based variability allows users to send messages, schedule appointments, make phone calls, find 
restaurants, use computing capabilities and cloud capability to enable individualized, context-relevant customer experiences. Cloud facilitates external collaboration with partners and customers, which can lead to productivity improvements and increased innovation. Cloud-based platforms can bring together disparate groups of people who can collaborate and share resources, information, and processes. A CC environment usually has multiple user terminals and service providers. From the collection terminals, the user collects the data using big data tools. On the other hand, the service provider saves, stores and processes big data. Therefore, CC provides a large data infrastructure. The infrastructure must provide resources and services on demand to ensure an uninterrupted flow. Because the cloud environment is scalable, it can provide an appropriate data management solution, regardless of the volume of data. Big data for data processing can be located globally, and maintaining such huge servers in different locations is an expensive measure for an organization. Because CC can store and process data by geographic dispersion and virtual servers, it significantly reduces the cost of processing big data. CC uses high-level software and applications that do not depend on the efficiency of the user's devices. In addition, it depends on the network servers and their power. On the contrary, if we use personal resources for big data, they will depend on the user's device.

At present, it is amazing to see the lack of automation tools in the cloud security environment, especially from the perspective of the risk associated with erroneous configurations and the reduction of budgetary resources allocated to IT. The lack of automation and integration between the various IT tools used makes it considerably more difficult for resource-limited security teams to effectively secure cloud environments. The lack of a centralized and global perspective on information from security tools is the biggest challenge to the optimal management of network security tools. The use of automated network security tools, robust application programming interface (API) structures, and integrations between hybrid and public cloud environments could help security teams take real-time control of their work environments. companies, minimizing the challenges posed by manual processes, the complexity of the infrastructure and the lack of visibility. It could also help offset budget cuts in the area of IT and the growing chronic lack of data security expertise.

There are still a number of barriers that make it difficult to adopt the cloud, especially in highly regulated industries. Even in the financial sector, the fragmentation of regulations affects the adoption of the cloud within the organization due to the large number of rules. In this regard, data location restrictions and cross-border inconsistencies (different interpretation and application of surveillance guidelines and requirements) continue to act as key barriers to optimal cloud adoption. This is especially true for multinational organizations, which want to generate a holistic view of their global business and risk profile and can benefit from services in different regions from cloud service providers to enable greater operational resilience. In addition to the main barriers mentioned above, there are other factors that need to be considered to highlight the full potential of CC. Traditional outsourcing rules address the risks arising from reliance on a third party for the provision of essential services. The main concern is that a firm may be unable to perform essential tasks or provide services if a third party fails. Such outsourcing rules impose restrictions and protect the use of cloud service providers. The benefits of redundancy, scalability and resilience mitigate the risk of continuity, but this is not fully understood by all stakeholders. Another such barrier is the interdependence between the cloud and old systems.

Migrating to CC is a challenge, many organizations prioritize new applications that will be deployed in the cloud, while old applications remain in old systems. This interdependence can create problems during the process and can be a major barrier if not managed properly. A good migration to CC is a source of new capabilities and security for any organization in relation to a traditional architecture. However, if not done correctly, it can turn into a major source of vulnerabilities. Having the right people in the organization that commands this process is of paramount importance, and access to the real small market for software engineers can be a huge barrier for some organizations. Today, data is generated in a large volume and it is difficult to store it for sure. Most companies need a place to store data securely. So many companies are adopting CC that it is estimated that cloud providers will offer more data centers at a lower price because there is a lot of competition between them.

In this context, the performance of the Internet will be greatly improved. Based on IoT and CC we can store data in the cloud, for analysis and to provide improved performance to applications. Users expect high quality fast charging services and applications. IoT is also one of the most important technologies because it comes with a continuous innovation in real-time data analysis. There are many machine-to-machine communication links, data, and processes that we can easily do with CC. The modular software will be rethought. The size of an individual program along with the complexity increase regularly. This leads to the fact that cloud technology will soon require an advanced thinking of computing systems. We can see the development of software from several angles, because in the future the applications will be stored in other locations. This can also reduce the cost of software because placing program components on different storage spaces is economical. Companies use a lot of software, which has not yet been modified. This leads to the fact that CC requires modified software to provide better security. This software will be easier and more flexible to use. One of the major advantages of this software will be that it will save both the overall cost and time. Data stored in the cloud will be analyzed using a machine and will not require any human help. The pace of 
project implementation in cloud environments exceeds the ability to adjust infrastructure in an efficient manner. This in itself is a challenge, as the number of providers and locations that need to be secured for the effective protection of infrastructure is constantly growing. The use of two or more public cloud platforms further increases complexity and affects visibility across the entire infrastructure. At the same time, the reduction of IT budgets is an additional factor that generates gaps in the overall data protection of the infrastructure used. The number of organizations worldwide spending less than a quarter of the total security budget on cloud protection has increased in the last year. Last but not least, as attack areas and the potential for fraud have expanded as the use of cloud media has expanded, many organizations have reduced their security teams in numbers, reducing their effectiveness, increasing the stress they face members of these teams and increasing the potential for human error.

Organizations can use the cloud in combination with IoT, AI, Fog and Edge Computing to improve current products and services, customer experiences, to retain and attract new customers, earning incremental revenue. Cloud can help a company create new products and services, use new channels or payment methods to attract existing or adjacent customer segments, in an attempt to generate significant new revenue. Cloud adoption can help an organization maintain its place in an existing value chain through increased efficiency and an improved capacity for partnership and collaboration. By assisting in the development of new operating capabilities, the cloud can help a company change its role in its industry or enter a different industry. Cloud business facilitators are already driving innovation in value propositions for customers and in the value chains of the company and industry. Businesses apply cloud computing to generate additional revenue streams by improving, expanding, and inventing new value propositions for customers, improving, transforming, and creating new value chains for organizations and industries. This has led to changes in who creates value, as well as how this value is created, delivered and captured. Thus, cloud computing accelerates industry dynamics, disrupts existing business models and fuels digital transformation.

\section{Conclusions}

CC has become a critical IT infrastructure for almost every aspect of everyday life and will continue to transform the world we live in on many levels and in different ways, along with IoT, Big Data, Artificial Intelligence or Cognitive Systems. The Internet of Things (IoT) has emerged to digitize our daily tasks in various systems, for example, smart homes, smart cities, smart factories, smart grids, and smart healthcare. Because cloud systems provide computing infrastructure, power, bandwidth, software, platforms, and storage, IoT applications integrate with cloud systems. IoT networks include communications from sensors, actuators, and services, which require high computing resources to run big data analytics applications and cybersecurity applications. Cloud systems suffer from a lack of support for mobility, latency, location awareness and geo-distribution. Fog / Edge paradigms have been proposed to enhance the benefits of cloud systems and to allow big data analysis at the edge of the network. A technique has been developed for connecting an intelligent data communication and pre-processing module to Cloud-IoT networks. The technique integrated a smart gateway with a Fog computing technique to reduce the computing area from the cloud. Some fog computing capabilities were used to develop an intrusion detection system for recognizing cyber attacks on wireless sensor networks. Policy-based management has been proposed to protect collaboration and interoperability between the different requirements of Fog node customers. A physical security framework needs to be developed to integrate the functions of the IoT, Fog and Cloud systems or a framework for identifying harmful activities at the edges of the network. Organizations that are concerned about risk and compliance (regulated financial services firms) will launch a private cloud, which is provided for exclusive use by a single organization comprising multiple consumers or business units. It may be owned, managed and operated by the organization, a third party or a combination thereof and may exist on or off the premises. In a community of common interests, within local administrations, health communities, a community cloud can be implemented. A key advantage of the public cloud infrastructure as a service (IaaS) and as a PaaS platform, respectively, is to place applications closer to customers to allow a better user experience. Execution of tasks in the private cloud is also exploited, especially where intellectual property, control, regulation or compliance issues, security, performance and cost of service are differentiated. Private clouds run on-premises, in roommate facilities or in provider facilities and can provide similar benefits to public CC. A modern cloud environment or cloud application consists of multiple cloud providers and thus integrates multiple microservices or platform services from different providers using a multi cloud model. In this model, a company develops most of its environment or applications and expands the architecture with other external services, whose efforts would be too great to develop them internally from scratch. The developed cloud supply chain is an important part of a digital infrastructure platform for any business and must be taken into account to benefit from the horsepower capacity of cloud infrastructure, platforms and applications. The hybrid cloud market is extremely competitive and consists of several important players. These companies use strategic collaborative initiatives to increase their market share and increase profitability. Companies operating in the market also purchase start-ups that work on hybrid cloud technologies to strengthen their product capabilities. 


\section{References}

1. Ahmed M., Litchfield A.T. (2018): Taxonomy for identification of security issues in cloud computing environments. Journal of Computing Information Systems, 58 (1): 79-88

2. Annette J. R., Banu W. A., Chandran P. S. (2015): Rendering-as-a-service: Taxonomy and Comparison. Procedia Computer Science, 50:276-281

3. Bayramusta M., Nasir V. A. (2016): A fad or future of IT?: A comprehensive literature review on the cloud computing research. International Journal of Information Managament, 36 (4): 635-644

4. Benlian A., Hess T. (2011): Opportunities and risks of Software-as-a-Service: findings from a survey of IT executives. Decision Support Systems, 52(1): 232-246

5. Benlian A., Kettinger W. J., Sunyaev A., Winkler T.J. (2018): Special section: The transformative value of cloud computing: a decoupling, platformization, and recombination theoretical framework. Journal of Managament Information Systems, 35(3): 719-739

6. Bottum J., Atkins D., Blatecky A., McMullen R., Tannenbaum T., Cheetham J., Wilgenbusch J., Bhatia K., Deumens E., B. von Oehsen, Fox G., Ziolkowski M., Bedrossian A., and Fay D., (2017): The Future of Cloud for Academic Research Computing. Technical Report of the 2016 National Science Foundation-funded workshop "Cloud https://www.researchgate.net/publication/317835476_The_Future_of_Cloud_for_Academic_Research_Computing

7. Burda D., Teuteberg F., (2014): The role of trust and risk perceptions in cloud archiving - results from an empirical study. J. High Technol Manag Res 25(2):172-187

8. Cafaro M., Aloisio G. (2011): Grids, clouds, and virtualization. In: Cafaro M, Aloisio G (eds) Grids, clouds and virtualization. Computer Communications and Networks. Springer, London, pp 1-21

9. Cloudscene S., (2018): Top ten data center operators in North America, EMEA, Oceania and Asia for the January to March 2018 period. https://cloudscene.com/top10, Accessed 7 Nov. 2020

10. Dašić P., Dašić J., Crvenković B., (2016): Service models for cloud computing: search as a service (SaaS). International Journal Engineering Technology, (IJET) 8(5): 2366-2373

11. ENISA (2012) Cloud computing - benefits, risks and recommendations for information security. European network and security agency. https://resilience.enisa.europa.eu/cloud-security-and-resilience/publications/cloud-computing-bene-fits-risks-and-recommendationsfor-information-security. Accessed 29 Oct. 2020

12. Hentschel R., Leyh C., Petznick A., (2018): Current cloud challenges in Germany: the perspective of cloud service providers. Journal of Cloud Computing, 7(1): 5

13. Hussain S.A., Fatima M., Saeed A., Raza I., Shahzad R.K., (2017): Multilevel classification of security concerns in cloud computing. Applied Computing Information, 13(1): 57-65

14. Kunz I., Stephanow P., (2017): A process model to support continuous certification of cloud services. Paper presented at the 31st IEEE international conference on advanced information networking and applications, Taipei, 27-29 Mar 2017

15. Lins S., Schneider S., Sunyaev A., (2018): Trust is good, control is better: creating secure clouds by continuous auditing. IEEE Transaction in Cloud Computing, 6(3): 890-903

16. Marston S., Li Z., Bandyopadhyay S., Zhang J., Ghalsasi A., (2011): Cloud computing - the business perspective. Decision Support Systems, 51(1): 176-189

17. Pahl, C. (2015): Containerization and the PaaS cloud. IEEE Cloud Computing, 2(3):24-31

18. Park S.T., Park E. M., Seo J.H., Li G. (2016): Factors affecting the continuous use of cloud service: focused on security risks. Cluster Computing, 19(1): 485-495

19. Parviainen P., Tihinen M., Kääriäinen J., Teppola S. (2017): Tackling the digitalization challenge: how to benefit from digitalization in practice. Int. J. Inf. Syst. Proj. Manag.5(1),63-77, https://doi.org/10.12821/ijispm050104

20. Rexford J., Balazinska M., Culler D., and Wing J., (2018): Enabling Computer and Information Science and Engineering Research and Education in the Cloud. Technical Report. Arlington, VA: National Science Foundation. https://dl.acm.org/citation.cfm?id=3233928

21. Ross J.W., Sebastia, I.M., Beath C.M. (2017): How to develop a great digital strategy. MIT SloanManag. Rev.58(2), 7-9

22. Schneider S., Sunyaev A. (2016): Determinant factors of cloud-sourcing decisions: reflecting on the IT outsourcing literature in the era of cloud computing. Journal of Information Technology, 31(1):1-31

23. Schneider S., Wollersheim J., Krcmar H., Sunyaev A. (2018): How do requirements evolve over time? a case study investigating the role of context and experiences in the evolution of enterprise software requirements. Journal of Information Technology, 33(2):151-170

24. Sharma D., Dhote C., Potey M.M. (2016): Identity and access management as security-as-a-service from clouds. Procedia Computer Science, 79: 170-174

25. Singh A., Chatterjee K., (2017): Cloud security issues and challenges: a survey. Journal of Network Computing Applications, 79(C): 88115

26. Trenz M., Huntgeburth J., Veit D. (2018): Uncertainty in cloud service relationships: uncovering the differential effect of three social influence processes on potential and current users. Information Managament 55(8):971-983

27. Yousef A. M. Qasem, Rusli Abdullah, Yusmadi Yaha and Rodziah Atana (2020): Continuance Use of Cloud Computing in Higher Education Institutions: A Conceptual Model, Department of Software Engineering and Information System, Faculty of Computer Science and Information Technology, University Putra Malaysia, Serdang 43400, Malaysia; Published: 23 September 2020, Appl. Sci.2020,10, 6628; doi:10.3390/app10196628

28. (2016): National Academies of Sciences, Engineering, and Medicine. Future Directions for NSF Advanced Computing Infrastructure to Support U.S. Science and Engineering in 2017-2020. Washington, DC: The National Academies Press. https://doi.org/10.17226/21886

29. (2018): National Institutes of Health. NIH Strategic Plan for Data Science." https://datascience.nih.gov/nih-strategic-plan-data-science

30. (2019): The White House. Executive Order 13859 on Maintaining American Leadership in Artificial Intelligence. https://www.whitehouse.gov/presidential-actions/executive-order-maintaining-american-leadership-artificial-intelligence/The focus here is specifically on the use of commercial cloud computing services for AI R\&D. The development of agency-hosted cloud services for $R \& D$ and other services is being considered elsewhere, notably at https://cloud.cio.gov/strategy/

31. Anwar Ghani, Afzal Badshah, Saeed Ullah Jan, Abdulrahman A. Alshdadi and Ali Daudhttps, (2020): Issues and challenges in Cloud Storage Architecture: A Survey. Received: 1 April 2020;nRevised: 7 Jun 2020; Accepted: 8 Jun 2020; Published Online: 10 June 2020, Researchpedia Journal of Computing, Volume 1, Issue 1, Article 6, Pages 50-65, Jun 2020//arxiv.org/pdf/2004.06809.pdf 